\title{
Characterizing the Switching Thresholds of Magnetophoretic Transistors
}

\author{
Roozbeh Abedini-Nassab, Daniel Y. Joh, Melissa A. Van Heest, John S. Yi, Cody Baker, \\ Zohreh Taherifard, David M. Margolis, J. Victor Garcia, Ashutosh Chilkoti, \\ David M. Murdoch, and Benjamin B. Yellen*
}

Single cell arrays are an increasingly important tool for biology, ${ }^{[1-10]}$ however, most existing single cell platforms are unable to perform large-scale operations on single cells with the flexibility and automation of modern day computer circuits. Inspired by the random access memory (RAM) architecture, we developed a novel approach for logic-based transport of single cells in microfluidic environments. ${ }^{[11]}$ This approach uses passive circuit elements (conductors, capacitors, and diodes) to transport single cells to pre-designated switching junctions. Active circuit elements (transistors and multiplexers) are used to switch the direction of cells at junctions, enabling their individual isolation, multiplexing, and retrieval from the array. The switching thresholds of previous transistor architectures, however, have yet to be experimentally quantified. Moreover, prior systems lacked reciprocal writing capabilities (i.e., ability to import a cell into, and export a cell out of, an array site). Finally, surfactants were required to prevent adhesion of particles and cells to the chip surface, which limited its capability for extended cell cultures.

Here, we experimentally quantify for the first time the switching thresholds of a general three-terminal magnetophoretic transistor. Our efforts to optimize the device for low-power consumption, along with the development of novel biocompatible surface coatings, have enabled a novel platform which can be scaled to address biologically relevant problems in single cell analysis.

\author{
R. Abedini-Nassab, C. Baker, Z. Taherifard, \\ Prof. A. Chilkoti, Prof. B. B. Yellen \\ Department of Mechanical Engineering and \\ Materials Science \\ Duke University \\ Box 90300 Hudson Hall, Durham, NC 27708, USA \\ E-mail: yellen@duke.edu \\ D. Y. Joh, Prof. A. Chilkoti, Prof. B. B. Yellen \\ Department of Biomedical Engineering \\ Duke University \\ Durham, NC 27708, USA \\ M. A. Van Heest, Dr. J. S. Yi, Dr. D. M. Murdoch \\ Department of Medicine \\ Duke University \\ Durham, NC 27708, USA \\ Prof. D. M. Margolis, Prof. J. Victor Garcia \\ Division of Infectious Diseases, Center for AIDS Research \\ Department of Medicine \\ University of North Carolina at Chapel Hill \\ Chapel Hill, NC 27599, USA
}

DOI: 10.1002/adma.201502352
The magnetophoretic transistor device has strong parallels with the electronic bandgaps of conventional three-terminal transistor switches. We create a semiconducting region for cell transport by introducing a small gap between two magnetizable disks (Figure 1a,b). This gap prevents the crossing of single cells and beads, except in the presence of an external gate bias. The transport properties can be understood through the spacetime variations in the magnetic potential energy landscape. The energy is determined by computing the local magnetic field distribution $\vec{B}(\vec{r})$, and by assuming that the beads and cells behave like point dipoles with magnetic moments $\vec{m}=\chi V \vec{B} / \mu_{0}$, where $V$ is the volume, $\chi$ is the volumetric magnetic susceptibility of the beads or cells, and $\mu_{0}$ is the magnetic permeability of vacuum. The energy landscape in the horizontal plane located at one cell radius above the substrate is calculated from: $U=-(1 / 2) \vec{m} \cdot \vec{B}$. As shown in Figure 1a,b, a double-well potential exists in the absence of a gate current with the energy barrier located at the center of the gap (see Figure 1a,b). This energy barrier prevents spontaneous cell and bed motion across the gap in the unactuated state. However, if the magnetic field is locally tuned by an adjacent microwire (gold wire of Figure 1a), then the double well potential merges into a single energy minimum, allowing the cells to be transferred across the gap. The current threshold required to activate particle transport across the gap is similar to the band referenced Fermi level of semiconductors. The direction of particle crossing (toward or away from the microwire) can be controlled with the field orientation and the direction of the applied gate current.

We designed, optimized, and built magnetophoretic transistors from aligned layers of magnetic permalloy disk patterns and gold wire patterns. These devices were fabricated on silicon wafers (University Wafer, Boston, MA) by conventional photolithographic lift-off technique. The magnetic pattern consisted of a $5 \mathrm{~nm} / 100 \mathrm{~nm}$ bilayer stack of $\mathrm{Ti} / \mathrm{Ni}_{81} \mathrm{Fe}_{19}$. The overlaid microwire patterns consisted of a $5 \mathrm{~nm} / 100 \mathrm{~nm}$ bilayer stack of $\mathrm{Ti} / \mathrm{Au}$. In between each metallic layer and on the top and bottom surfaces, we deposited $250 \mathrm{~nm}$ of $\mathrm{SiO}_{2}$ by plasmaenhanced chemical vapor deposition (PECVD, Advanced Vacuum Vision 310) in order to electrically isolate the gate electrodes from the magnetic layer and fluid. More information on the processing conditions and experimental testing protocol is provided in Materials and Methods (Supporting Information).

The transistor geometry consists of permalloy disks with radius, $R$, and thickness, $\tau_{\mathrm{m}}$, which are separated by a gap distance, $d$, at the switching junction. A metallic wire of thickness, $\tau_{\mathrm{w}}$, is overlaid with its left edge shifted by a distance, $\lambda$, from the disk edge on the opposing side of the gap. Locally strong 


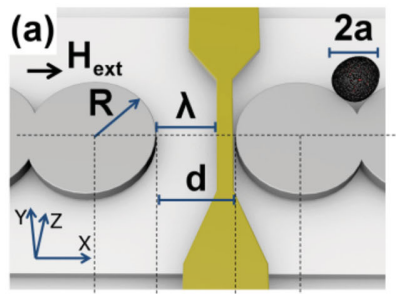

(b)
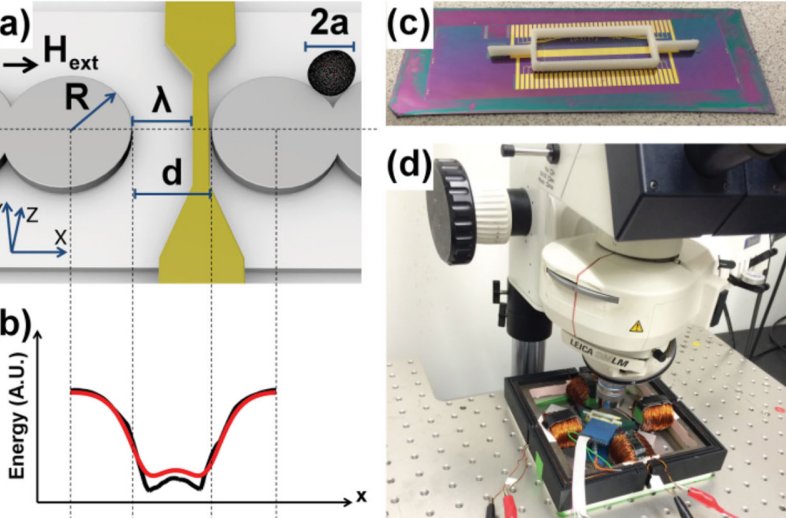

Figure 1. Illustration of the transistor geometry and potential energy distribution. a) $\mathrm{Ni}_{81} \mathrm{Fe}_{19}$ permalloy disks (gray circles) of radius, $R$, and thickness, $\tau_{\mathrm{m}}$, are separated by a small gap with separation distance, $d$. A gate electrode (gold wire) is overlaid with its left edge shifted from the opposing gap side by a distance, $\lambda$. A magnetized cell (black circle) of radius, $a$, is shown moving along the magnetic track. The external field direction, $H_{\text {ext }}$, is aligned along the track axis, depicted as the black arrow. b) The potential energy line cross section is simulated at a vertical height of $z=a$ from finite element (FEM) analysis (black line), and compared with an analytical charge model (red line) based on Equations $1 \mathrm{a}$ and $1 \mathrm{~b}$. c) A photograph of the chip and 3D printed container used to test the transistors. d) The overall instrument apparatus.

magnetic fields are created by narrowing the wire to a width, $r_{\mathrm{w}}$, at the junction. Magnetically labeled cells are depicted by the black circle, having a mean radius, $a$.

We used finite element (FEM) simulations (Ansys, Maxwell 15.0) to compute the magnetic fields in the gap between two disks, when in the presence of a uniform magnetic field applied along the track axis ( $x$-direction). The equivalent magnetic charge distribution was evaluated on the disk surface using the relation: $\sigma=\left(\vec{H}_{\text {in }}-\vec{H}_{\text {out }}\right) \cdot \hat{n}$. The charge density on the axial surfaces and the perimeter are denoted by $\sigma_{\mathrm{s}}$ and $\sigma_{\mathrm{p}}$, respectively. Their geometric variations were found to correspond to hyperbolic and periodic functions as

$\sigma_{\mathrm{s}}(r, \theta)=\sigma_{0 \mathrm{~s}} \sinh \left(\gamma \frac{r}{R}\right) \cos (\theta)$

$\sigma_{\mathrm{p}}(\theta)=\sigma_{\mathrm{op}} \cos (\theta)$

where the best fitting parameters were found to be $\sigma_{0 \mathrm{p}}=$ $6.2 H_{\text {ext }}, \sigma_{0 \mathrm{~s}}=0.12 H_{\text {ext }}$, and $\gamma=4$.

Once the charge distributions are determined, the magnetic potential $\phi_{\mathrm{m}}$ is calculated by integrating over these charged surfaces

$\phi_{\mathrm{m}}\left(\vec{r}_{\mathrm{s}}\right)=\oiint \frac{\sigma\left(\vec{r}_{\mathrm{s}}\right)}{4 \pi\left|\vec{r}-\vec{r}_{\mathrm{s}}\right|} \mathrm{d} S$

The fields and field gradients produced by the disks are then calculated by taking successive derivatives of the magnetic potential, ${ }^{[12]}$ which allows for computation of the magnetic forces and potential energies. This method was used to produce energy line cross sections (red line, Figure 1b), which
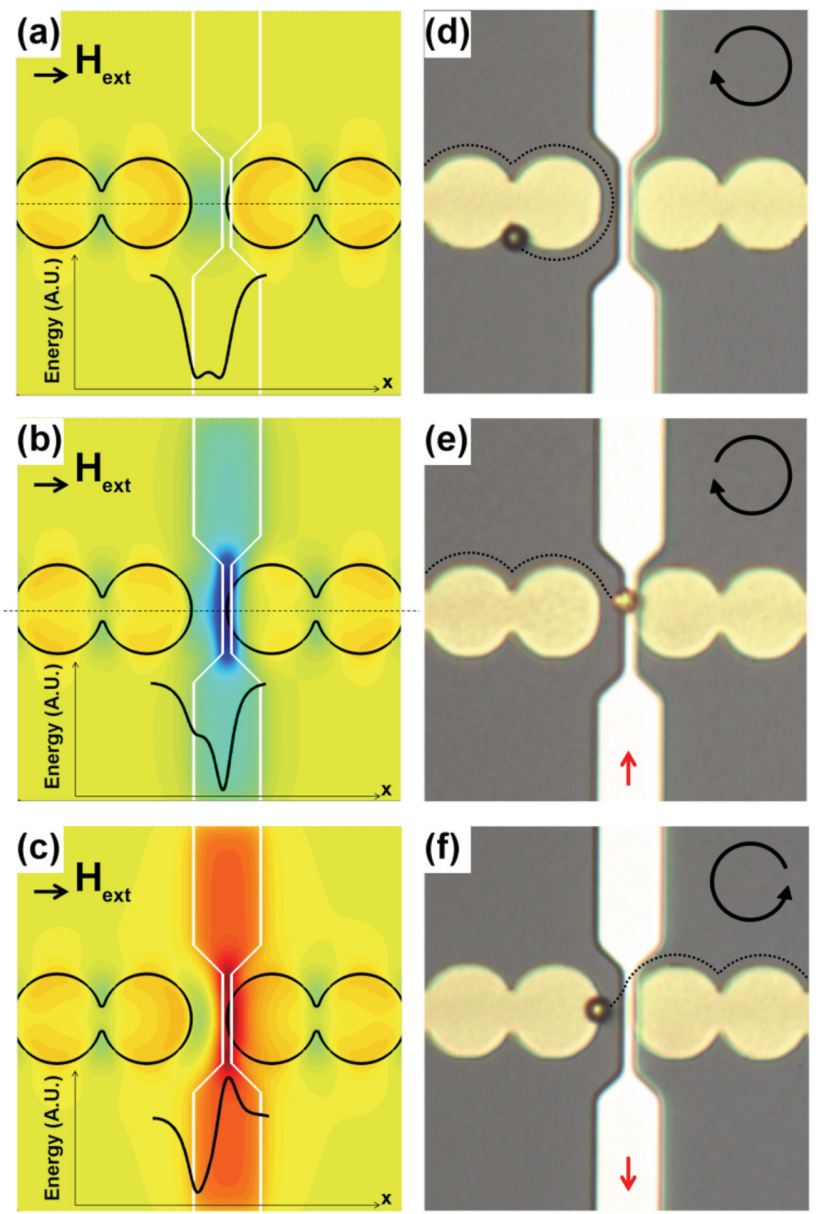

Figure 2. Transistor operational modes and their energy landscapes. Magnetic potential energy landscapes are shown in $(a-c)$ with corresponding experiments shown in $(\mathrm{d}-\mathrm{f})$. The line cross sections are shown a) when no gate current is applied, b) in the attractive mode, and c) in the repulsive mode. In all cases, a constant uniform magnetic field is applied along the positive $x$-direction and denoted by the black arrow. d) The bead remains on the same side of the gap due to the double well potential of part (a). e) In a clockwise rotating field, the bead moves across the gap toward the wire due to a gate current in the positive $\gamma$-direction. f) In a counter-clockwise rotating field, the bead crosses the gap and moves away from the wire due to a gate current in the negative $\gamma$-direction. The field rotation sense is denoted by the circular arrows, and the gate currents are shown as red arrows.

are in good agreement with the FEM based results (black line, Figure $1 \mathrm{~b})$. The energy surface cross sections at one cell radius above the substrate are also shown in Figure 2, where the blue regions denote the local energy minima. The magnetic field from the gate electrode is calculated with the Biot-Savart law, assuming an infinitely thin sheet current

$\vec{B}_{\text {wire }}(\vec{r})=\frac{\mu_{0}}{4 \pi} \iint \frac{\vec{K}\left(\vec{r}_{\mathrm{s}}\right) \times\left(\vec{r}-\vec{r}_{\mathrm{s}}\right)}{\left|\vec{r}-\vec{r}_{\mathrm{s}}\right|^{3}} \mathrm{~d} S$

where the wire's field is assumed to be produced by a sheet current, $\vec{K}\left(r_{\mathrm{s}}\right)$ which is a reasonable approximation when the wire thickness $(\approx 100-200 \mathrm{~nm})$ is much smaller than the minimum planar dimensions of the wire $(\approx 2-3 \mu \mathrm{m})$. In order to simplify 
our theoretical model, we assumed that the fields from the wire and magnetic disks are non-interacting. These assumptions were deemed necessary for computational efficiency to analyze switching properties and particle trajectories.

One of the important challenges we faced stems from the tendency of cells to avidly adhere to untreated surfaces. Nonspecific cell adhesion hinders controlled manipulation and must be solved for proper functioning of these multiplexed devices. To address this limitation, we deposited a "nonfouling"protein and cell resistant-poly(oligoethylene glycol methyl methacrylate) (POEGMA) $50 \mathrm{~nm}$ thick coating on top of the chip surface that reduced cell adhesion to the substrate. POEGMA surfaces were deposited by surface-initiated atom transfer radical polymerization (SI-ATRP). The approach has been previously described, ${ }^{[13]}$ but has never been attempted on lithographically patterned substrates. Briefly, the POEGMA layer was constructed by first functionalizing the $\mathrm{SiO}_{2}$ top surface of the chip with ATRP initiator and then immersing the substrates in polymerization solution, producing surface-tethered brushes of POEGMA. In order to protect the exposed electrodes and connectors, a 3D-printed chamber was then fastened on the chip with silicone adhesive, shown in Figure 1c.

Magnetic beads (Spherotech CM 50-10) with mean diameters of $5.7 \mu \mathrm{m}$ were used to test the transistor performance in deionized water. Magnetically labeled human CD4+ T-cells of a similar size range were also tested by conjugation to anti-CD4 antibody labeled magnetic nanoparticles (StemCell Technologies, Vancouver, Canada). The labeling efficiency and cell isolation purity were confirmed by flow cytometry. We developed a custom stage to perform microscopy, while also allowing for the application of rotating magnetic field. This stage, shown in Figure 1d, consists of a four-pole iron plate (McMaster Carr) wrapped with magnet wire (20AWG), and powered by programmable power supplies (BOP 20-5M, Kepco, Flushing, NY). We made electrical contact to the gate electrodes with IC test clips (Ponoma Electronics, Everett, WA). Both the rotating fields and gate currents were controlled by customized LabVIEW programs (National Instrument), and the transport experiments were monitored in a Leica DM LM microscope using a Retiga 2000R video camera (Figure 1d).

We investigated two types of transistor modes, including an attractive mode, shown in Figure $2 \mathrm{~b}, \mathrm{e}$, and a repulsive mode shown in Figure 2c,f. The energy landscapes of each of these modes in the presence and absence of gate currents are shown in Figure 2a-c. Sufficiently strong gate currents can annihilate the double well energy barrier of Figure 2a, and induce the cell to transfer across the gap toward the wire (attractive mode) or away from the wire (repulsive mode). Example particle trajectories for each transistor mode are provided in Figure $2 \mathrm{~d}-\mathrm{f}$, where the corresponding external field directions and in-plane rotating fields are also illustrated.

We measured the switching thresholds of the attractive and repulsive transistor modes through a combination of static and dynamic transistor tests, with the results shown in Figures 3 and 4. From simulations, we expected that optimal transistor performance can be achieved when the gap distance is similar to the cell diameter. ${ }^{[14]}$ To test this hypothesis, we fabricated a series of transistors with gap distances ranging from 5 to $15 \mu \mathrm{m}$ to cover a range of dimensions relative to the typical diameters of human T-cells $(6-10 \mu \mathrm{m})$. For transistors gaps smaller than $8 \mu \mathrm{m}$, we observed a high frequency of particle crossings in the absence of a gate current, rendering this geometry unsuitable for a transistor switch. For transistors gaps larger than $10 \mu \mathrm{m}$, we found that excessively large gate currents were required to achieve reliable switching. The rest of this analysis thus focused on gap dimensions of 8 or $10 \mu \mathrm{m}$, which is slightly larger than the average cell diameter.

We investigated two approaches for evaluating the transistor performance, referred to as "static" and "dynamic" transistor tests. In the static test, we positioned the cells or beads at the gap junction, and then aligned the external field along the track 
axis ( $x$-direction). Next, we slowly increased the gate current, and recorded hysteresis loops for the position versus current, and in the process identified the gate currents when the cells and beads transferred across the gap (see Movies S1 and S2, Supporting Information, for beads and for cells, respectively). The switching experiments were conducted on at least ten beads or cells for each experimental condition, and we repeated the experiment in triplicate for each specific bead or cell. The average switching current thresholds for the attractive and repulsive transistor modes for magnetic beads and cells, respectively, are shown in the bar plots of Figures 3c,d and 4c,d.

In order to compare these experimental results with our theoretical model, we computed the expected current switching thresholds, assuming that the bead diameter was a random variable, with mean and standard deviation of $5.72 \pm 0.86 \mu \mathrm{m}$, as derived from pixel analysis based size measurements of a population of 30 magnetic beads. The simulation results and their standard deviations for the switching thresholds are provided in Figure 3e,f, and used for comparison with experiments on magnetic beads. Simulations for cells show similar trends (data not shown).

In the bar plots of Figures 3 and 4, each bar corresponds to a specific transistor geometry with a unique gap size and gate location. The black, red, and blue bars correspond to transistors with a gap size of $10 \mu \mathrm{m}$, but with different offsets for the wire displacement from the disk edge, ranging from 7 to $9 \mu \mathrm{m}$. The green bars of Figures 3 and 4 correspond to transistors with a smaller gap size of $8 \mu \mathrm{m}$ and a displacement of the microwire by $6.5 \mu \mathrm{m}$ from the gap center. In all cases, the wire width kept fixed at $3 \mu \mathrm{m}$, which is the smallest photolithographically reproducible dimension.

Though there are some differences between theory and experiment, the simulations results predict the correct order of magnitude of the required switching currents, shown to be in the range of 10-30 mA. Experiments also confirmed our predictions that magnetic beads will switch at lower current threshold when the gap size is smaller. This trend is clearly observed in the repulsive transistor modes of Figure 3c,e; however, the trend still appears to be consistent for the attractive transistor modes and for the cell based switching experiments of Figure 4. Some features predicted in the theory were not observed in experiment, which are attributed to the oversimplifying assumptions in our idealized theoretical model. Improving the simulation accuracy would require better knowledge of the magnetic domain structure in the disk patterns as a function of the applied magnetic field, as well as more rigorous treatment of the field distribution using finite element models.

We next employed dynamic testing of the transistor switching efficiency, which is more representative of the device operating conditions. Using the transistor geometry of $d=10 \mu \mathrm{m}$ and $\lambda$ $=9 \mu \mathrm{m}$, the number of successful bead and cell crossings were
Repulsive Mode
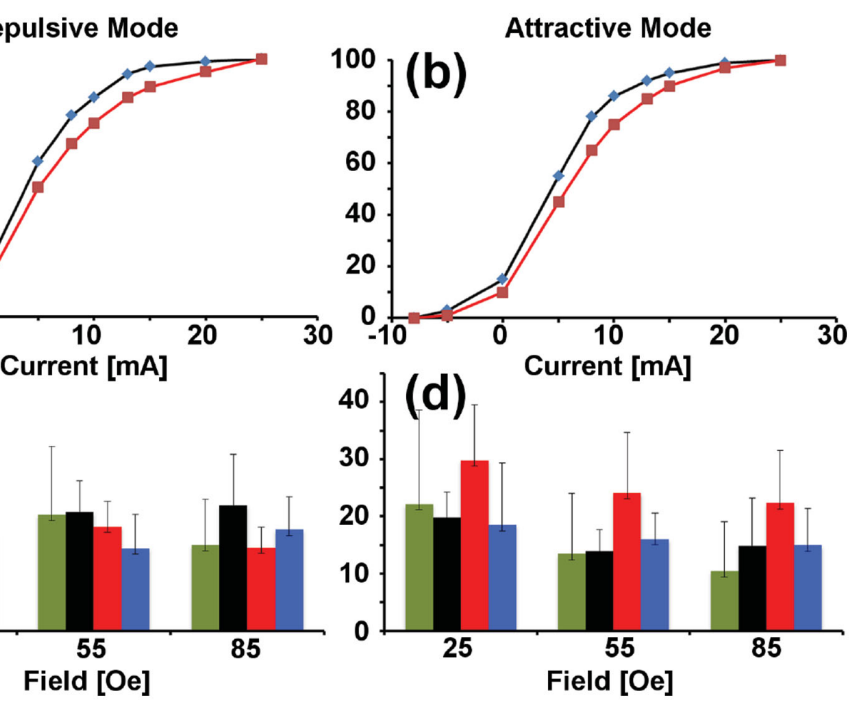

Field [Oe]

monitored as a function of the applied gate currents in an externally applied 45 Oe rotating magnetic field. The switching efficiency is also shown as a function of frequency for single beads in Figure 3a,b and for single cells in Figure 4a,b. The motion of the beads and cells in the dynamic transistor tests are also shown in Movies S3 and S4 (Supporting Information).

In comparing the data for the static and dynamic tests, we found that at low frequencies the switching thresholds were commensurate, which validates the simpler static tests to quantify transistor performance. At higher frequencies, the switching thresholds are reduced, as previously predicted, ${ }^{[14]}$ and thus frequency can be used as an additional control parameter to reduce the switching thresholds. For the magnetic beads in the repulsive mode (Figure 3a), we observe complete switching with gate currents of $\approx 8 \mathrm{~mA}$ for the 0.5 and $0.8 \mathrm{~Hz}$ driving frequencies. On the other hand, complete switching is achieved with gate currents of $\approx 15 \mathrm{~mA}$ when the driving frequency is $0.2 \mathrm{~Hz}$, which is consistent with the static transistor tests. For the cell data, we find that complete switching is achieved at gate currents of $\approx 20-25 \mathrm{~mA}$ for the 0.2 and $0.5 \mathrm{~Hz}$ driving frequencies, which is also consistent with the static transistor tests.

The ability to achieve both attractive and repulsive modes in a single transistor architecture allows us to implement the full write cycle (i.e., writing a bead or cell to the array, and extracting it from the array). These dual functions are depicted in Figure 5, where the red lines show the trajectories of magnetic beads being written to array sites, and the green lines show the trajectories of beads being retrieved from array sites. In total, this demonstration required nine synchronized switch operationsthree row switches were activated to introduce cells down specific rows in the array, while three column switches were activated in the repulsive and attractive mode, in order to import 


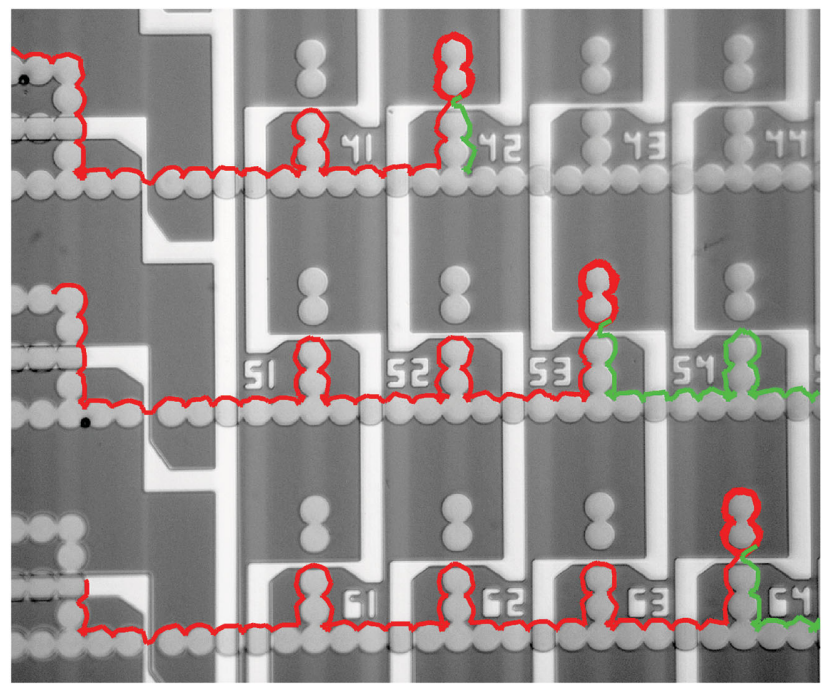

Figure 5. Multiplexed arrays. An $8 \times 8$ array was designed and built to import, store, and export magnetized objects. A small section of the array is shown here, in which three magnetic beads are temporarily stored in array sites 42, 53, and 64 with the import trajectories shown in red. At later times, the beads are exported from these storage sites with the trajectories shown in green. The full trajectories can be found in Movie S5 (Supporting Information).

or export the beads, respectively, from their designated storage sites. The actual trajectories are shown in Movie S5 (Supporting Information), and an additional high-resolution Movie S6 (Supporting Information) demonstrates that similar operations can be performed on magnetized cells.

The use of passive circuit elements (magnetic tracks) to transport magnetized objects in parallel with an external rotating magnetic field, in combination with active circuit elements (transistors) to switch objects at specific junctions, provides a scalability that is similar to that found in a computer RAM memory. As such, this platform avoids potential issues with localized heating and high power densities of nDEP and optical systems $^{[15-19]}$ and allows large numbers of objects to be controlled with relatively few gate electrodes. Our experimental quantification of the switching thresholds in three-terminal magnetophoretic transistor devices thus establishes the baseline for future efforts to scale this tool into a novel, biologically relevant platform for single cell analysis.

\section{Supporting Information}

Supporting Information is available from the Wiley Online Library or from the author.

\section{Acknowledgements}

The authors are thankful to Cheol Gi Kim, of DGIST, Daegu, South Korea, for helpful discussions, and to Hamidreza Toroghi for providing the illustration in Figure 1a. This research was supported by the Creative and Novel Ideas in HIV Research Program (CNIHR) through a supplement to the University of Alabama at Birmingham (UAB) Center for AIDS Research (CFAR) funding (P30 A1027767). This funding was made possible by collaborative efforts of the Office of AIDS Research, the National Institutes of Allergies and Infectious Diseases, and the International AIDS Society. This study was also supported in part by $\mathrm{NIH}$ grants 1R56Al112360 (Murdoch), Al096113, and Al111899 (Garcia) and by the NIH funded CFARs at Duke University and University of North Carolina - Chapel Hill (5P30 Al064518 and Al50410).

Received: May 17, 2015

Revised: July 27, 2015

Published online: September 9, 2015

[1] N. E. Navin, Genome Biol. 2014, 15, 452

[2] Ch. Ma, R. Fan, H. Ahmad, Q. Shi, B. Comin-Anduix, Th. Chodon, R. C. Koya, Ch. Liu, G. A. Kwong, C. G. Radu, A. Ribas, J. R. Heath, Nat. Med. 2011, 17, 738.

[3] J. Kim, D. Taylor, N. Agrawal, H. Wang, H. Kim, A. Han, K. Rege, A. Jayaraman, Lab Chip 2012, 10, 1813

[4] A. Mullard, Nat. Rev. Drug Discovery 2011, 10, 477.

[5] P. K. Chattopadhyay, T. M. Gierahn, M. Roederer, J. Ch. Love, Nat. Immunol. 2014, 15, 128

[6] R. Satija, A. K. Shalek, Trends Immunol. 2014, 35, 219.

[7] B. Gaudillière, G. K. Fragiadakis, R. V. Bruggner, M. Nicolau, R. Finck, M. Tingle, J. Silva, E. A. Ganio, C. G. Yeh, W. J. Maloney, J. I. Huddleston, S. B. Goodman, M. M. Davis, S. C. Bendall, W. J. Fantl, M. S. Angst, G. P. Nolan, Sci. Transl. Med. 2014, 6, 255 ral31.

[8] V. Chokkalingam, J. Tel, F. Wimmers, X. Liu, S. Semenov, J. Thiele, C. G. Figdor, W. T. S. Huck, Lab Chip 2013, 13, 4740.

[9] M. Wu, A. K. Singh, Curr. Opin. Biotechnol. 2012, 23, 83.

[10] S. Lindstrom, H. Andersson-Svahn, Lab Chip 2010, 10, 3363.

[11] B. Lim, V. Reddy, X. Hu, K. Kim, M. Jadhav, R. Abedini-Nassab, Y. Noh, Y. T. Lim, B. B. Yellen, C. Kim, Nat. Commun. 2014, 5, 3846.

[12] J. D. Jackson, Classical Electrodynamics, Wiley, New York, NY 1998.

[13] A. Hucknall, D. H. Kim, S. Rangarajan, R. T. Hill, W. M. Reichert, A. Chilkoti, Adv. Mater. 2009, 21, 1968.

[14] R. Abedini-Nassab, D. M. Murdoch, C. G. Kim, B. B. Yellen, J. Appl. Phys. 2014, 115, 244509.

[15] N. Mittal, A. Rosenthal, J. Voldman, Lab Chip 2007, 7, 1146.

[16] K. R. Love, S. Bagh, J. Choi, J. C. Love, Trends Biotechnol. 2013, 31, 280.

[17] P. Y. Chiou, A. T. Ohta, M. C. Wu, Nature 2005, 436, 370.

[18] J. P. Frimat, M. Becker, Y. Y. Chiang, U. Marggraf, D. Janasek, J. G. Hengstler, J. Franzke, J. West, Lab Chip 2011, 11, 231.

[19] S. Lindstrom, H. Andersson-Svahn, Lab Chip 2010, 10, 3363. 